\title{
NOUVELLE
}

\section{Autophagie et destruction des lymphocytes T CD4 par le VIH-1}

Lucile Espert, Mélanie Denizot, Marina Grimaldi, Véronique Robert-Hebmann, Bernard Gay, Mihayl Varbanov, Patrice Codogno, Martine Biard-Piechaczyk

L. Espert, M. Denizot, M. Grimaldi, V. Robert-Hebmann, B. Gay, M. Varbanov, M. Biard-Piechaczyk :

Laboratoire Infections Rétrovirales et Signalisation cellulaire, UMR 5121CNRS/UMl, Institut de Biologie,

4, boulevard Henri IV, 34000 Montpellier, France.

P. Codogno: Inserm U756, Faculté de Pharmacie, Université Paris XI, 92296 Châtenay-Malabry, France. lucile.espert@univ-montpl.fr
> La mort cellulaire programmée (PCD, programmed cell death) est un processus physiologique essentiel pour le maintien de l'homéostasie. Au cours de l'évolution, plusieurs mécanismes de mort, souvent intriqués, se sont mis en place pour assurer au mieux la survie des organismes [1]. L'absence de PCD peut contribuer au développement de pathologies telles que le cancer et, de façon inverse, une PCD excessive peut aussi devenir pathologique, comme c'est le cas pour l'immunodéficience provoquée par le VIH-l (virus de l'immunodéficience humaine). Cela montre bien la nécessité d'un très haut niveau de régulation entre survie et mort cellulaire. Deux types majeurs de PCD sont connus: la PCD de type I ou apoptose et la PCD de type II, ou mort autophagique $[2,3]$.

L'autophagie (Figure 1) est un mécanisme physiologique hautement régulé dépen-

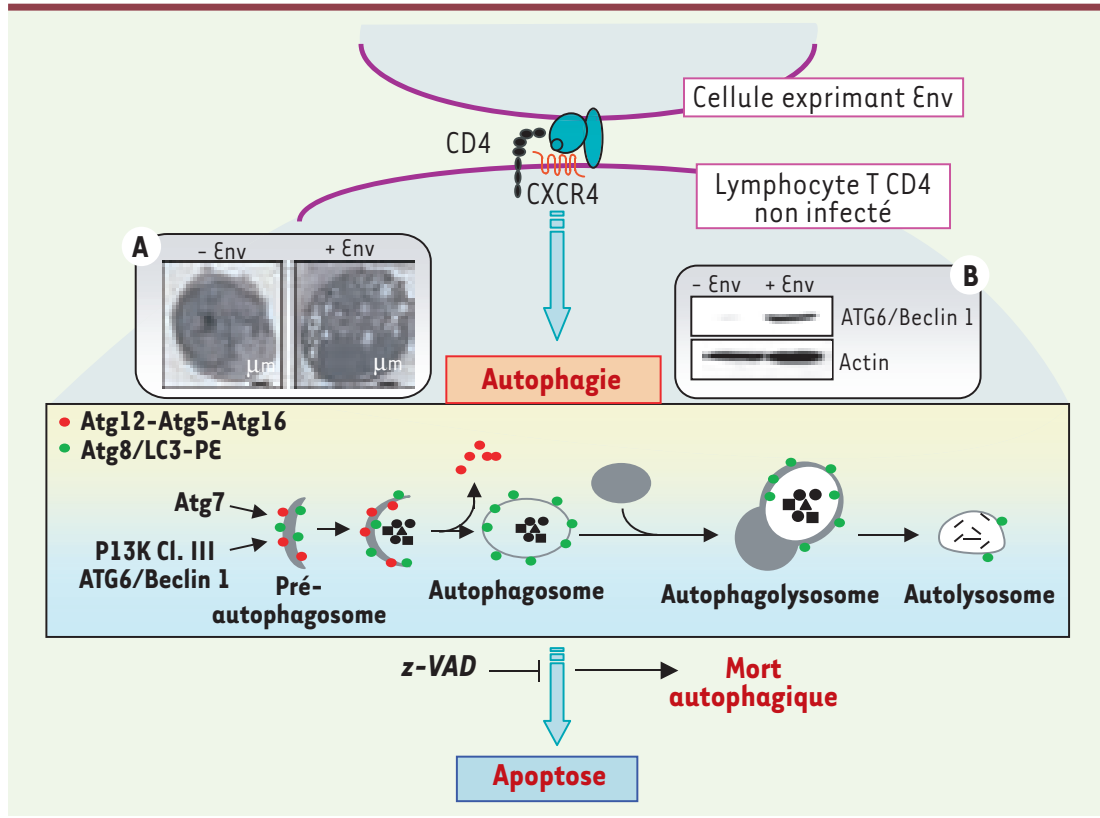

Figure 1. L'autophagie est nécessaire au déclenchement de l'apoptose des lymphocytes T CD4 non infectés après la liaison des glycoprotéines d'enveloppe du VIH-1 sur le co-récepteur CXCR4. Lorsque l'apoptose est inhibée, on observe une mort de type autophagique. A. Microscopie électronique montrant l'accumulation d'autophagosomes dans le cytoplasme des lymphocytes T CD4 non infectés après liaison de Env sur CXCR4. B. Western-blot montrant l'accumulation de la protéine autophagique ATG6/Beclin 1 dans les lymphocytes T CD4 non infectés après liaison de Env sur CXCR4.

dant des gènes ATG (autophagy-related genes), faisant intervenir deux systèmes de conjugaison semblables au processus d'ubiquitination (formation des conjugués Atg12-Atg5-Atg16 et Atg8/LC3-PE), et de plusieurs voies de signalisation [4]. La découverte des gènes ATG chez la levure a permis de reconsidérer ce processus longtemps laissé de côté par manque d'outils [5]. L'autophagie a pour fonction primaire de réguler le recyclage des protéines à durée de vie longue et des organelles cellulaires. Ce processus est caractérisé par une dégradation de composés cytoplasmiques à l'intérieur de vacuoles appelées autophagosomes. Les autophagosomes fusionnent avec les lysosomes pour former des autophagolysosomes qui sont les compartiments terminaux de la dégradation [4]. La phosphatidylinositol 3 -kinase de classe III est impliquée dans les phases précoces de la formation des vacuoles et contrôle la voie autophagique en s'associant avec la protéine ATG6/ Beclin 1 au niveau du réseau trans-golgien [6]. Les mécanismes moléculaires impliqués dans la formation des autophagosomes et régulés par ce complexe de signalisation sont encore indéterminés. L'autophagie est un processus complexe car il peut conduire soit à la survie, soit à la mort cellulaire [7].

De façon intéressante, de plus en plus de travaux montrent que l'autophagie et l'apoptose sont des processus étroitement liés. En effet, l'autophagie peut être indépendante de l'apoptose, l'inhiber ou 
au contraire être nécessaire à son induction. Cependant, les voies de signalisation responsables de ces connexions ne sont pas claires [8]. Un lien moléculaire entre ces deux processus provient de l'interaction entre la protéine autophagique ATG6/Beclin 1 et la protéine anti-apoptotique $\mathrm{Bcl}-2$. Cette interaction pourrait jouer un rôle de «rhéostat» entre autophagie et apoptose et ainsi déterminer le devenir de la cellule $[9,10]$.

\section{Mort cellulaire et infection par le VIH-1}

L'infection par le VIH-l entraîne une destruction progressive des lymphocytes T CD4 aboutissant, à plus ou moins long terme, à la phase Sida (syndrome d'immunodéficience acquise). De nombreux travaux ont permis de montrer que des phénomènes d'apoptose survenant dans des lymphocytes T CD4 infectés, mais aussi et surtout dans les lymphocytes T non infectés, étaient responsables, au moins en partie, de la disparition de cette population cellulaire [11]. La mort des cellules T CD4 peut être induite par des interactions directes entre le virus et la cellule cible et par l'activation des systèmes immunitaires et inflammatoires de l'hôte. Parmi les déterminants viraux impliqués dans ce phénomène de mort cellulaire, les glycoprotéines d'enveloppe virales (gp120/gp4l, ici appelées Env pour enveloppe) jouent un rôle majeur en interagissant avec le récepteur CD4 et un corécepteur, CXCR4 ou CCR5, molécules de signalisation intervenant dans la physiologie cellulaire [12]. Malgré de nombreux travaux réalisés, la nature des premiers signaux induits directement par le virus et conduisant à la mort cellulaire n'a toujours pas été identifiée.

Les résultats obtenus dans notre équipe montrent que l'interaction entre $\varepsilon$ nv et CXCR4 active une voie de signalisation conduisant à la voie intrinsèque d'apoptose (relargage de cytochrome $\mathrm{c}$ de la mitochondrie, activation des caspases 9 puis 3 ), et ainsi à la mort des cellules T CD4 par apoptose sans infection ni réplication virale [13]. Cependant, les expériences montrent que la mort cellulaire induite par Env n'est pas totalement bloquée lorsque l'apoptose est inhibée par un inhibiteur général des caspases (z-VAD). Cette observation nous a conduit à rechercher le rôle de l'autophagie dans ces phénomènes de mort cellu- laire induits par $\varepsilon$ nv. Très récemment, nous avons montré que l'interaction entre Env, exprimée à la surface de cellules, et CXCR4 induit également un processus autophagique dans les cellules T CD4 non infectées (Figure $1 \mathrm{~A}$ ). Nous avons également observé une accumulation de la protéine ATG6/ Beclin 1 (Figure 1B). Fait remarquable, par des techniques d'interférence par l'ARN (ciblant spécifiquement l'expression des protéines ATG7 et ATG6/Beclin 1), ainsi que par l'utilisation d'inhibiteurs chimiques (3-méthyl-adénine et bafilomycine Al), nous avons montré que l'autophagie était un processus nécessaire au déclenchement de l'apoptose induite par Env. De plus, lorsque l'apoptose est bloquée par l'utilisation de zVAD, la mort cellulaire n'est pas totalement inhibée, suggérant qu'il existe également un processus de mort autophagique (PCD type II) [14]. Les signaux de mort activés dans une cellule T CD4 induits après liaison de Env semblent d'un niveau de complexité élevé. La découverte de la régulation de ces signaux est un grand défi scientifique pour les années à venir. L'implication de l'autophagie est une première avancée importante dans ce domaine.

\section{Perspectives}

Il est de plus en plus évident que la modulation de l'apoptose ou de l'autophagie dans la cellule hôte par les agents pathogènes joue un rôle important dans les pathologies associées. Les pathogènes peuvent réguler l'expression de protéines anti-apoptotiques afin d'éviter le déclenchement de l'apoptose tant qu'ils ne se sont pas assez multipliés dans la cellule hôte. Ils peuvent également réguler la réponse autophagique, soit pour engendrer des lieux de réplication plus efficaces, soit pour induire une mort cellulaire et permettre leur dissémination [15, 16]. Il apparaît donc essentiel de progresser dans la compréhension des mécanismes moléculaires impliqués dans la régulation des voies apoptotique et autophagique au cours d'infections par des pathogènes.

La caractérisation précise des voies de signalisation impliquées dans l'autophagie, puis l'apoptose, nous permettrait, à terme, de découvrir de nouvelles cibles et donc de proposer de nouveaux outils pharmacologiques. Les progrès obtenus ces dernières années dans le traitement du cancer, à l'aide de molécules agissant sur ces voies de signalisation sont encourageants dans ce sens. L'étude des mécanismes de mort cellulaire programmée devient incontournable afin de progresser dans la compréhension du maintien de l'homéostasie et de l'intégrité cellulaire. $\diamond$

Autophagy and CD4 T lymphocyte

destruction by HIV-1

\section{REMERCIEMENTS}

Ce travail a été soutenu par le Centre National de la Recherche Scientifique (CNRS), I'Université Montpellier I (UMI), Sidaction et l'Agence Nationale de Recherche sur le sida (ANRS).

\section{RÉFÉRENCES}

1. Golstein P, Kroemer G. Redundant cell death mechanisms as relics and backups. Cell Death Differ 2005; 12 (suppl 2): 1490-6.

2. Edinger AL, Thompson CB. Death by design : apoptosis, necrosis and autophagy. Curr Opin Cell Biol2004 ; $16: 663-9$.

3. Lockshin RA, Zakeri Z. Apoptosis, autophagy, and more. Int J Biochem Cell Biol 2004 ; 36 : 2405-19.

4. Marino G, Lopez-Otin C. Autophagy : molecular mechanisms, physiological functions and relevance in human pathology. Cell Mol Life Sci 2004 ; 61 : 1439-54.

5. Codogno P. Les gènes ATG et la macrophagie. Med Sci (Paris) $2004 ; 20: 734-6$.

6. Kihara A, Kihara A, Kabeya Y, et al. Beclinphosphatidylinositol 3-kinase complex functions at the trans-Golgi network. EMBO Rep $2001 ; 2$ : 330-5.

7. Codogno, P, Meijer AJ. Autophagy and signaling : their role in cell survival and cell death. Cell Death Differ 2005; 12 (suppl 2) : 1509-18.

8. Levine B, Yuan J. Autophagy in cell death : an innocent convict? J Clin Invest 2005; 115 : 2679-88.

9. Pattingre S, Tassa A, Qu X, et al. Bcl-2 antiapoptotic proteins inhibit Beclin 1-dependent autophagy. Cell 2005 ; $122: 927-39$.

10. Pattingre S, Levine B. Bcl-2 inhibition of autophagy : a new route to cancer? Cancer Res $2006 ; 66: 2885-8$.

11. Petit F, Petit F, Arnoult D, et al. Intrinsic and extrinsic pathways signaling during HIV-1 mediated cell death. Biochimie 2003; 85 : 795-811.

12. Alimonti JB, Ball TB, Fowke KR. Mechanisms of $\mathrm{CD} 4^{+} \mathrm{T}$ lymphocyte cell death in human immunodeficiency virus infection and AIDS. J Gen Virol 2003 ; 84 : 1649-61.

13. Roggero R, Robert-Hebmann V, Harrington $S$, et al. Binding of human immunodeficiency virus type 1 gpl20 to CXCR4 induces mitochondrial transmembrane depolarization and cytochrome c-mediated apoptosis independently of Fas signaling. J Virol $2001 ; 75: 7637-50$.

14. Espert L, Denizot M, Grimaldi M, et al. Autophagy is involved in T cell death after binding of HIV-1 envelope proteins to CXCR4. J Clin Invest 2006; 116: 2161-72.

15. Wileman T. Aggresomes and autophagy generate sites for virus replication. Science 2006 ; 312: 875-8.

16. Ogawa M, Sasakawa C. Bacterial evasion of the autophagic defense system. Curr Opin Microbiol $2006 ; 9$ : 62-8. 